\title{
Case Reports in the Korean Journal of Family Medicine
}

\author{
Jungun Lee
}

Department of Family Medicine, Sanbon Medical Center, School of Medicine Wonkwang University, Gunpo, Korea

Besides original articles, other types of manuscripts in the Korean Journal of Family Medicine (KJFM) include review articles, brief communications, editorials, letters to the editor-inchief, and case reports. Recently our editors discussed the case report guidelines of the KJFM.

A case report by other specialists was submitted. Many family physicians were unfamiliar with this case, however, it was well known within its specialty area and was previously reported several times in other medical journals. They argued that the case would be instructive in differentiating other common diseases from the symptoms described. After some debate we made the decision to reject this case primarily because the patient directly visited specialists in a tertiary hospital, and was treated without the involvement of any family doctors or primary care physicians.

The information for authors of KJFM describes about prerequisites for case reports as this. ${ }^{1)}$ 'A case example should be one that has never been observed up to the present time and has never been reported before. If the case example is determined to be significant enough, although it may relate to a very common condition or a description that may not be appropriate to delineate, decision to accept the article shall be determined following the review of the editorial committee.'
Considering that most of our readers are working in the primary care field, there are many interesting cases from other specialties which are similar to this case. Even if it is related with common symptoms, we thought primary care physicians should contact the patients first during the course. In addition, brief communication is another example that needs more detailed guidelines.

\section{CONFLICT OF INTEREST}

No potential conflict of interest relevant to this article was reported.

\section{REFERENCE}

1. Korean Journal of Family Medicine. Information for authors [Internet]. Seoul: Korean Journal of Family Medicine [cited 2014 Feb 4]. Available from: http://www.kjfm.or.kr/file/ $\mathrm{pdf} / \mathrm{kjfm}$ _information_for_authors.pdf. 